\title{
Minimal invasive beating heart tricuspid valve repair in the presence of a severely calcified ascending aorta
}

\author{
Mahmut Ay*, Andreas Zierer and Thomas Schachner \\ Department of Cardiothoracic and vascular surgery, Kepler University hospital Linz, Austria
}

\begin{abstract}
This is a case report about a minimal invasive beating heart tricuspid valve repair in the presence of a severely calcified ascending aorta in an adult patient. Especially in the presence of severe aortic calcification it was the better choice to operate in beating heart technique. We reported the case with CT scan and intraoperative situs graphics.
\end{abstract}

\section{Introduction}

The tricuspid valve disease is for a long period of time asymptomatic. Dyspnoea, ascites, hepatosplenomegaly, pleural effusions and peripheral edema are typical symptoms of progressive disease. Combined with compromised ejection fraction and other comorbidities, patients are in a poor clinical condition. Many of the tricuspid valve operations are combined with other left ventricular valve interventions. Isolated tricuspid valve surgery can be performed on arrested heart or beating heart. Especially in the presence of severe aortic calcification it can be the only choice.

\section{Case report}

A 69 year old man with COPD in poor clinical condition with severe tricuspid valve insufficiency was presented to our hospital with dyspnoea on effort graded as NYHA functional class grade III. A right ventricular decompensation was 2 months ago. After lasix therapy he lost $5 \mathrm{~kg}$ of fluid. Still he had pretibial edema. The electrocardiogram revealed atrial fibrillation; laboratory tests showed restricted haemoglobin $9.2 \mathrm{~g} / \mathrm{dl}$, the other parameters were normal. NT pro BNP was $656.2 \mathrm{pg} / \mathrm{ml}$. The chest radiograph showed right sided mediastinal dilatation and signs of pulmonary obstruction.

The preop echocardiography revealed biatrial and right ventricular dilatation with mildly reduced right ventricular function. LVEF was $55 \%$ with an asynchronicity of septal movement. Tricuspid insufficiency with grade $4^{\circ}$ was present, Vena contracta was $1.7 \mathrm{~cm}$, the tricuspid annulus was $48 \mathrm{~mm}$, other valves showed no relevant pathologies.

The CT scan revealed severe atherosclerosis of the aorta. On the ventral side there were few non-calcified areals. So we decided to perform a minimal-invasive operation on the beating heart.

Anesthesia was given as for standard cardiac procedures. A central venous line was applied via the right internal jugular vein. After median sternotomy and incision of the pericardium we palpated massive aortic calcification. A small spot could be found for the aortic cannulation but aortic cross clamping appeared to be high risk for neurologic injury or aortic damage. We decided to perform the valve intervention on the beating heart. After bicaval cannulation, snaring of the SVC and IVC was accomplished we started cardiopulmonary bypass and gained adequate drainage.
Atriotomy was performed down to the IVC cannula in an oblique fashion. After placement of the pledgeted traction sutures, the valve was examined. The tricuspid valve pathology consisted of severe annular dilatation (Type I). Pulmonary hypertension was quantified with a pulmonary artery pressure of $47 \mathrm{mmHg}$ (systolic).

Tricuspid annuloplasty was performed using a $34 \mathrm{~mm}$ MC3 Ring (Edwards Inc.). The operative course and weaning from the heart lung machine was uneventful. The patient was extubated on postoperative day 1. Initial respiratory insufficiency (due to COPD and pulmonary hypertension) improved during the following days. The further hospital stay was uncomplicated and the patient was discharged home on pod 17. The postop echocardiography showed residual regurgitation 1st grade plus. The pulmonary artery pressure was estimated with $35-40$ mmHg. Ejection fraction and other parameters were stable as pre-op (Figures 1 and 2).

\section{Discussion}

In the presence of annular dilatation and normal valve leaflets is our primary goal to repair the tricuspid valve. The beating heart and minimalinvasive approach via upper sternotomy in the presented case
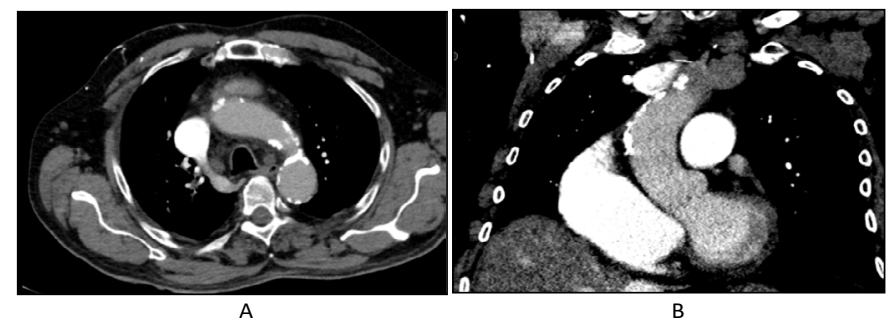

Figure 1. Preoperative CT scan showed a severely calcified ascending aorta and aortic arch

Correspondence to: Mahmut Cetin Ay, Department of Cardiothoracic and vascular surgery, Kepler University hospital Linz, Austria, Tel: +43576808378436; Fax: +4357680832178; E-mail: mahmut.ay@kepleruniklinikum.at

Key words: tricuspid valve disease, Dyspnoea, CT scan

Received: June 20, 2017; Accepted: July 07, 2017; Published: July 10, 2017 


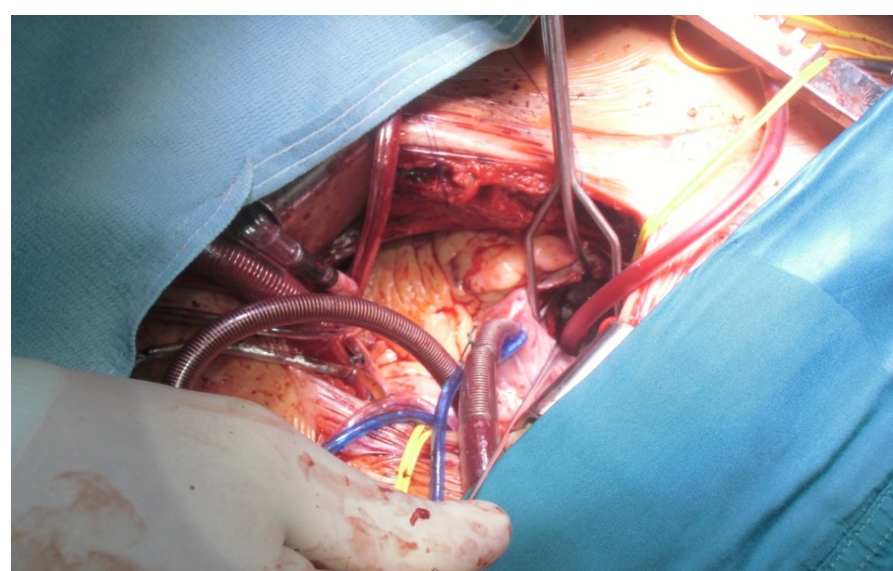

Figure 2. Intraoperative situs for beating heart tricuspid valve repair via partial upper sternotomy.

was feasible. A crossclamping of the aorta would have meant a high risk for aortic injury and severe neurologic deficites.

In the literature the following risk factors for a failure of tricuspid repair are described: lleft ventricular ejection fraction $<35 \%$ and the need for permanent pacemaker wires through the tricuspid valve [1].

In most cases of tricuspid regurgitation a repair can be achieved. However, some pitfalls should be considered. The main attention should be payed to the conductance system during the placement of the sutures for the septal leaflet: Too deep bites could cause AV Block
[2]. In addition wrong stitches in the area of the anterior leaflet can cause aortic valve dysfunction.

Previous studies showed no significant differences in the perioperative outcome and 30-day mortality if we compare beating heart to arrested heart tricuspid valve surgery [3]. Even though in general beating heart surgery showed no superiority over arrested heart surgery, it is the better choice in the presence of severe ascending aortic atherosclerosis.

The limited access via partial upper sternotomy preserved the integrity of the lower thorax, improved respiratory recovery and provided a better cosmetic result compared with full sternotomy. Early mobilisation of the patient was possible.

\section{Conclusion}

Successful minimal invasive beating heart tricuspid valve repair is an attractive option in the presence of severe ascending aortic atherosclerosis.

\section{References}

1. McCarthy PM, Bhudia SK, Rajeswaran J, Hoercher KJ, Lytle BW, et al. (2004) Tricuspid valve repair: durability and risk factors for failure. J Thorac Cardiovasc Surg 127: 674-685. [Crossref]

2. Ohata T, Kigawa I, Yamashita Y, Wanibuchi Y (2000) Surgical strategy for severe tricuspid valve regurgitation complicated by advanced mitral valve disease: long-term outcome of tricuspid valve supra-annular Implantation in eighty-eight cases. $J$ Thorac Cardiovasc Surg 120: 280-283. [Crossref]

3. Baraki H, Saito S, Al Ahmad A, Fleischer B, Haverich A, et al. (2015) Beating heart versus arrested heart isolated tricuspid valve surgery. Int Heart J 56: 400-407. [Crossref]

Copyright: (2017 Ay M. This is an open-access article distributed under the terms of the Creative Commons Attribution License, which permits unrestricted use, distribution, and reproduction in any medium, provided the original author and source are credited. 